\title{
Foreword: On Grids and Networks
}

The "grid" and the "network" have become pivotal notions in a wide variety of areas of research and creation. The present volume constitutes indisputable evidence of this trend. It is truly amazing that the same structural concepts are shared by such an abundance of fields as modern art, urban planning, the creator's role in aesthetics, music, computer-aided design and production, acting, cinema art, verbal interaction, and virtual reality. This collection of essays will most probably challenge many researchers to fine-tune the grid aspects common to the varied multiplicity of fields, and will open new venues to promote them. It corresponds, though not directly or too strictly, with the international conference entitled "The Multidisciplinary Grid 2020" that took place in November 2020 at HIT - Holon Institute of Technology (an institution of which both the Editors and I are members). The conference, organized by the Department of Multidisciplinary Studies, was aimed at exploring the "grid" as a cross-disciplinary theme. It strove to explore a new horizon of relationships and fusion of the "grids", and to foster a fruitful dialogue concerning gridded interpretations among researchers, practitioners, and artists. ${ }^{1}$

Although a distinction between the grid and the network may not be mandatory-and the present collection further blurs and softens the boundaries between them - it is still tempting to somewhat elaborate on potentially different emphases. These varied emphases, or focal points, may promote a better understanding of the foundations and the discovery of new potential directions, which might be of interest to experts in many diverse areas. The following remarks point to this path in conversation between exact sciences, applied science, fine arts, and humanities. As a mathematician, I admit to a slight and quite expected bias towards the first two of these areas.

Mathematicians seem to have started dealing with these structures in the seventeenth century, specifically through ideas expressed by Gottfried Wilhelm Leibniz -a world-renowned mathematician, contemporary of Isaac Newton. Many consider Leonhard Euler's 1736 paper, “The Seven Bridges of Königsberg” (which provided a systematic solution to a popular puzzle), to be the starting point of what is presently known as "Graph Theory." In the formal, abstract-cum-precise language of mathematics, a graph is a set of objects referred to as "vertices", together with a prescribed set of pairs from among these vertices, referred to as "edges." Many would realize this notion as a set of points in a plane with lines connecting a number of pairs from among those points. A "graph" is therefore an abstract notion: more than what is actually considered in a graph are the aspects that are not of issue; essentially, it has nothing to do with graphics. The location of vertices is not significant, and so are the geometrical properties - straight or curved - of the edges connecting some of them;

1 For a few more words about this conference see the next Acknowledgements section. 
only the set of vertices in itself, and the existence of edges between certain pairs of vertices, are of significance. Typical issues include the following: is there a "triangle" in the graph - namely, three vertices such that each two are connected by an edge? Or, how many triangles are there? Is there an efficient algorithm to find "cliques", that is, sets of vertices in which every pair is connected by an edge? (Note that a triangle is a clique of three vertices).

Naturally, this abstract notion of "graph" has materialized in many different familiar contexts. For example, a set of traffic junctions and the relevant connecting roads; or a set of communication switches and the direct connections between pairs; or a group of people - say, within the nobility of some historical entity - where an "edge" indicates a parent-child relation. Nevertheless, this clear terminology helps to distinguish between the different contexts of grids and networks.

A network provides the environment for investigating the performance and consequences of the relations between connected elements (vertices) in the set (graph) under study. For instance, through how many intermediate switches in a communication system a signal would have to pass in order to arrive from any given switch to another? Or, for pixels within an image, under a fixed definition of color "similarity", what is the average length of a chain of neighboring pixels in which any two consecutive ones have a "similar" color?

On the other hand, a grid - although similarly consisting of a set of elements does not address relations between these elements. Rather, it focuses on the fact that they have been selected from a much wider set, and on the manner in which they represent, in some definite sense, that specific wider set. Very often, the original set is a continuum and the selected elements form a finite sample that makes it possible to perceive, study, process, or understand said continuum by referring to the representing grid. For instance, when Thomas Alva Edison thought about presenting moving dynamic scenes to spectators away from the site, he understood that the living scene was a continuum and that he had no way of capturing it and presenting the same continuum elsewhere. Then he came across the brilliant idea that it would suffice to capture still images of the dynamic scene with a high enough frequency - say, thirty images per second - in order to present the sequence to the spectators: he understood that this finite sample of still images would suffice to create for the spectators the perception of visualizing the real dynamic scene. The finite but quite large sample of still images is a grid whose elements have been selected from a continuum in a manner that makes them an adequate representation of the original continuum scene. Similarly, an image on a computer screen is a grid consisting of a finite sample of pixels, each providing the color of a certain point in a continuous real scene - a finite collection that generates a continuous impression.

The resolution of the human eye makes this grid an adequate representation of the real continuous scene. Similarly, data obtained from a well-scattered set of meteorological measuring devices forms a grid that adequately represents the synoptic status of the area under observation. Likewise, the finite number of images captured 
by a medical CT instrument hopefully provides an understanding of the body part being examined. In all these examples, the grid serves the purpose of adequately representing a continuum by cleverly selecting a finite sample out of the latter. Studying grids has mainly to do with ways to select the grid members, analyze the representation adequacy, and come up with novel ideas for applying grids to achieve new, unprecedented insights about continua as perceived from those grids.

Towards the end of the nineteenth century a movement emerged among European painters (such as George Seurat, Camille Pissarro, and Paul Signac, to mention but a few) called pointillism, which examined the ability to create a rich visual impression by providing only points of color rather than filling whole areas with continuous coloring.

Echoing this idea, it is interesting to point out that the grid concept has played a significant role in applied mathematics for many decades. Applied mathematicians have paid much attention to continuous natural phenomena since early times. Archimedes, in the third century BC, went quite far in understanding hydrostatic and dynamic phenomena in fluids, as exposed in his book On Floating Bodies. In the ninth century AD, al-Biruni in Khwarazm and Bukhara together with other Moslem scientists of the period thoroughly studied the physics of fluids. In the sixteenth century, Leonardo da Vinci studied turbulent flows in great detail, brilliantly applying his results in his paintings. The dynamics of gases and fluids are very challenging computationally; the corresponding differential equations are complex and essentially hopeless. Therefore, much effort has been devoted to perform calculations on representing grids. The basic idea is to represent the continuous fluid using a sufficiently dense grid of points, each of which is accompanied by a set of physical data (e.g., location, velocity, pressure, and temperature) specific to it. Once the representative grid is created, the complicated hydrodynamics of the fluid are analyzed on it. In fact, a very significant portion of the currently known and applied physical and engineering knowledge has been achieved using this approach. An important variant in this field is the application of variable grids. The idea is to increase the density of grid points in zones where phenomena are more extreme or fast-changing, which allows a more thorough investigation of said phenomena, and to return to the previous lower density when the zone becomes less hectic.

The grid concept has not evaded the field of sound and music. For many decades now, sound has been transferred in communication systems not in full, but rather as a sequence of representative samples (for example, short time-intervals) taken from the original vocal flow. To illustrate this idea, suppose that every second is divided into 40 equal 25-millisecond time-segments. Subsequently, each 1-millisecond subinterval of each segment is allocated to one particular sound source - the same millisecond subinterval in each of the 40-time segments. Thus, the listener does not receive a continuous data stream, but rather 40 discrete 1-millisecond samples of the vocal information per second. Experience shows that in this way, the human ears and brain perceive the vocal information quite adequately. At the same time, this idea enables 
the transfer of 25 sound streams simultaneously on a single communication channel. The 25 sampled milliseconds actually form a grid: a finite representation of a continuum.

Another grid-related realization may be found in one of the musical streams that originated at the beginning of the twentieth century: the twelve-tone music. The idea was to impose the condition that every bar in a musical composition should include each of the twelve tones of the octave exactly once, and thus create an artistic impression. Some people would argue that this is exactly the realization of a grid: within the framework of infinitely many, or at least very numerous, possible note combinations, the composer must select individual combinations out of a defined finite set of allowed ones, while still striving to create an intended emotional impression. Apparently, this direction has not yet gained wide recognition - is it because about half of the human brain cortex is dedicated to vision, whereas only about two percent are dedicated to hearing?

All the above examples serve to establish the following observations: grids and networks are formal discrete structures; their members are extracted from real continuous (and therefore infinite) entities; a network serves to study the origin entity when relations among members are the main focus of interest, whereas grids are applied when the faithfulness of the representation of the original entity by the extracted sample (rather than the relations among members) is the main issue. Can this distinction be helpful in future developments in research and creation? Is there a promise in dynamical grids?

Many would say that artwork, of all sorts, is a purposeful organization of a medium with the aim of evoking an emotional impression. In this respect, the following, formally stated, challenge might be proposed: to what extent can the pieces of the organized medium under discussion be segmented, and to what extent can the emotional impression be subdivided, so that the artwork accomplishes its artistic mission in full? It is quite plausible that a deeper understanding of the roles of grids and networks may enhance future developments in many intellectual endeavors through the suitable implementation of the specific strengths of these two basic notions.

Adir Pridor

March 2021

Herzliya \& HIT - Holon Institute of Technology, Israel 